this edition Dr Apley has nevertheless managed to keep the reader up to date in most things, confining himself to essentials and emphasizing the important. General principles are well set out. Difficult subjezts such as the causes of floppy infant and ma!absorption are clearly classified.

All undergraduates and postgraduates (this includes family practitioners) should certainly read the first chapter with the quotation from James Spence. It puts the child in hospital in perspective but of course since Dr Apley, among others, has pioneered for better facilities for children in hospital things have improved.

Certain points that might need attention in future editions might include the dosages quoted for drugs for paediatric practice, where the basic adult dose is always listed before the paediatric dosage. One would also suggest that more emphasis might have been given to the range of normal values given for weight, height, and head circumference.

These are minor points in an excellent text which may be easily carried in the pocket, which will not be terribly lightened by the cost of just under $£ 5.00$.

\section{A Primer of Medicine (4th edn)}

By M. H. PAPPWORTH. Pp. 438. Butterworths: London, 1978. $£ 7.95$

The author has received wide acclaim as a teacher and this book is his own personal contribution to the practice of medicine. It is not a complete text book of medicine, but the emphasis is placed on the art of clinical examination and diagnosis. In an age of ever increasing sophisticated diagnostic techniques, it is refreshing to find pride of place given to the eliciting of physical signs at the bedside.

It is nearly 20 years since the 1st Edition was published and the fact that the book is now in its 4 th edition is recommendation itself. This edition shows a number of changes and has a rewritten chapter on Limbs and two new chapters on Medical Vocabulary and Dermatology.

The author has serious reservations about the coining of new medical terms and jargon and feels that there is a danger of doctors becoming illiterate. The point is well made, but perhaps a little overplayed.

The chapter on Skin Diseases is brief, but outlines basic descriptive patterns such as macules, papules and vesicles and then gives a short outline of specific skin diseases.
The rest of the book follows its previous format with chapters, amongst others, on History Taking, Fever, General Appearance, The Head and Facies, Opthalmology, The Neck and The Limbs. The Respiratory, Cardiovascular, Alimentary, Genito-Urinary and Nervous Systems are given chapters for each system. Each is excellent and the reviewer particularly liked the balance between anatomy, physiology and pathology outlined in the chapter on the Nervous System.

This book must find a place in the bookshelf of every undergraduate and postgraduate studying for higher examinations. It is well presented and full of good common sense. It cannot be recommended too highly.

Surveillance for the Prevention and Control of Health Hazards due to Antibiotic-resistant Enterobacteria

Report of a WHO Meeting. Technical Report Series 624. Pp. 54. World Health Organization, Geneva, 1978. Sw.fr. 6.00.

The spread of drug resistance among Gram-negative intestinal and related bacteria continues at an alarming rate. While outbreaks of untreatable typhoid, dysentery and other enterobacterial infections have been reported throughout the world, a particularly sinister recent trend has been the transmission of ' $R$-plasmids' from this group of bacteria to unrelated species, including the gonococcus and Haemophilus influenzae. Methods of investigating and controlling these problems are concisely reviewed in this recent Technical Report from the World Health Organization. Within the confines of 47 small pages, the text deals in a remarkably comprehensive manner with all the main issues of this complex subject. Antibiotic misuse in man and animals, and the role of food and water in the spread of resistance are usefully discussed, as are the procedures for epidemiological and laboratory surveillance, and data collection.

Although published very recently, the Report has occasion ally been overtaken by events. For example, the statement 'Resistance is very rarely seen in $V$. cholerae' is no longer true in several endemic areas. This, however, serves to emphasize the dynamic nature of the problem, and the Report can be highly recommended as an authoritative and generally upto-date accourt of this extremely important subject. 